\title{
Review: ipratropium bromide with $\beta$-agonists improves pulmonary function and reduces admissions to hospital in acute asthma
}

Rodrigo G, Rodrigo C, Burschtin O. A meta-analysis of the effects of ipratropium bromide in adults with acute asthma. Am J Med 1999 Oct;107:363-70.

QUESTION: In adults with acute asthma treated with $\beta$-agonists, does the addition of ipratropium bromide improve pulmonary function during the first 90 minutes of treatment and reduce the rate of admissions to hospital?

\section{Data sources}

Studies were identified by searching Medline (1978 to April 1999), Current Contents, Science Citation Index, review articles, bibliographies of relevant articles, and the register of the Medical Editors' Trial Amnesty and by contacting experts and the manufacturer of ipratropium.

\section{Study selection}

English language studies were selected if they were randomised, doubled blind, controlled trials (RCTs); patients ( $>16$ y of age) had acute asthma and were treated in an emergency department with $\beta$-agonists; pulmonary function was measured as a continuous variable; and sufficient data were available to calculate effect sizes.

\section{Data extraction}

Data were extracted on year and source of publication, country, number of patients, patient characteristics, dose and route of all medications, spirometric measures used, mean and standard deviation of spirometric values at baseline and after treatment, length of follow up, statistical analysis, admission rate, and adverse effects. 2 reviewers, blinded to authors and outcomes, independently assessed the quality of study methods (score 0.08 to 1.0 , with $\geqslant 0.7$ indicating high quality studies).

\section{Main results}

10 published RCTs involving 1483 patients (mean age $32 \mathrm{y}, 64 \%$ women) met the selection criteria. The mean quality score was 0.66 . The typical ipratropium dose was $0.5 \mathrm{mg}$ mixed with a $\beta$-agonist given soon after arrival in the emergency department. In 4 RCTs, a new dose was given within 60 or $120 \mathrm{~min}$, and in 2 RCTs, ipratropium was given twice (at 45 and $120 \mathrm{~min}$ ). The overall pooled effect size from 10 RCTs for pulmonary function was $0.14(95 \%$ CI 0.04 to $0.24, \mathrm{p}=0.008)$, which is equivalent to a $10 \%$ (CI 2\% to $18 \%$ ) increase in $\mathrm{FEV}_{1}$ or peak flow in the ipratropium group relative to the placebo group. The effect remained when only high quality studies were combined. The pooled results of 5 RCTs showed that ipratropium led to a greater reduction in the admission to hospital rate than did placebo $(\mathrm{p}=0.007)$ (table).

\section{Conclusion}

In patients with acute asthma treated with $\beta$-agonists, the addition of ipratropium bromide improves pulmonary function in the first 90 minutes of treatment and reduces the rate of admissions to hospital.

Source of funding: no external funding.

For correspondence: Dr C Rodrigo, Centro de Terapia Intensiva, Asociación Española 1 a de Socorros Mutuos, Bulevar Artigas 1465, Montevideo 11300,

Uruguay.

Ipratropium bromide v placebo for acute asthma treated with $\beta$-agonists*

\begin{tabular}{llll} 
Outcome & $\begin{array}{l}\text { Number of } \\
\text { studies }\end{array}$ & $\begin{array}{l}\text { Pooled odds ratio } \\
(95 \% \mathrm{Cl})\end{array}$ & NNT (CI) \\
\hline Admission to hospital & 5 & $0.62(0.44$ to 0.88$)$ & $18(11$ to 77$)$ \\
\hline
\end{tabular}

*Abbreviations defined in glossary. Length of follow up not reported.

\section{COMMENTARY}

Current recommendations for the treatment of asthmatic exacerbations advocate the use of short acting $\beta$-agonists with or without ipratropium bromide. ${ }^{12}$ An expert panel report suggested considering the use of anticholinergic agents in all patients with a severe exacerbation. ${ }^{2}$ These recommendations are based on studies that offer mixed conclusions about whether ipratropium and a $\beta$-agonist lead to a better response than do $\beta$-agonists alone. Although many studies have not shown that combination therapy has a statistically significant beneficial effect, these studies have consistently shown a slightly greater improvement in lung function with combination therapy than with $\beta$-agonists alone.

Although the overall pooled effect size in the well done review by Rodrigo et al was equivalent to an improvement in lung function of only $10 \%$, patients with severe obstruction showed a substantial improvement $(>25 \%)$ with ipratropium bromide. One could question the clinical significance of these findings because most of the studies used suboptimal doses of $\beta$-agonists; however, 2 studies used aggressive $\beta$-agonist therapy ( 3 doses of salbutamol every 20 minutes or continuously).

The effect of ipratropium on hospitalisation rates, which was evaluated in 5 RCTs, was more clinically important. This analysis found that the early administration of ipratropium with $\beta$-agonists reduced the admission rate by $38 \%$. Although these studies were not designed to evaluate the effect of ipratropium on admission to hospital rates and standardized criteria for deciding on admission or discharge were not used, a recent large RCT in children found that a multiple-dose ipratropium protocol led to a reduction in the hospitalisation rate similar to that in the study by Rodrigo et al. ${ }^{2}$ Another recent RCT in adults showed a substantial reduction in the length of hospital stay when ipratropium was added to $\beta$-agonist therapy in the first 36 hours after admission to hospital. ${ }^{3}$ Rodrigo and colleagues' review adds support to the current recommendations to treat patients who have substantial exacerbations of asthma with a combination of ipratropium bromide and $\beta$-agonists.

Jay Peters, MD University of Texas Health Science Center at San Antonio San Antonio, Texas, USA

1 National Asthma Education and Prevention Program.Bethesda, MD: U.S. Department of Health and Human Services; 1997. NIH publication no. 97-4051.

2 British Guidelines Coordinating Committee. Thorax 1997;52:S1-30

3 Qureshi F, Pestian J, Davis P, Zaritsky A. N Engl J Med 1998;339:1030-5. 